\title{
Existence and uniqueness of the solution of an integro-differential equation of the second order with boundary conditions
}

\author{
Azzam S. Younis \\ Department of Mathematics / College of Education \\ University of Mosul
}

Received

11 / 11 / 2008
Accepted

15 / 07 / 2009

الخلاصة

يتضمن البهث درلسة وجود وققارب الطل لظلم من المعادلات تكلملي مة -الفاض لية المالية

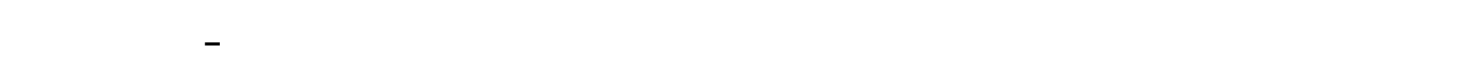
الل لظلم المعادلات الفاضلية اللاظطية ذاتشروط حدودية ل ـ Samoilenko A.M. كما قم من خلال هذا البحث تعميم الطريقة في أعلاه و توسيعها.

\section{Abstract}

In this paper we investigate the existence and approximation solution for nonlinear system of an integro-differential equation of the second order with boundary conditions by using the numerical-analytic method for investigating a system of nonlinear differential equation with boundary conditions which is given by Samoilenko A. M. And also these investigations lead us to generalized the above method.

\section{Introduction:}

The numerical- analytic method has been used to study many boundary value problems [1,2,3,4,5,7].

Samoilenko, A. M. and Ronto, N.I. used the numerical- analytic method for investigating a system of nonlinear differential equation with boundary conditions:

$$
\begin{aligned}
& \frac{d x}{d t}=f(t, x) \\
& A x(0)+C x(T)=d
\end{aligned}
$$

Where the vector function $\mathrm{f}(\mathrm{t}, \mathrm{x})$ is continuous in $\mathrm{t}, \mathrm{x}$ on the domain:

$$
(t, x) \in[0, T] \times D
$$

and $\mathrm{D}$ is a compact subset of the Euclidean spaces $R^{n}, A=\left(A_{i j}\right)$, $C=\left(C_{i j}\right)$, are positive matrices $(\mathrm{n} \times \mathrm{n}), d \in R^{n}$. 
In this paper we use the numerical-analytic method of a system of nonlinear differential equation which was given by Samoilenko, A. M. Consider the following problem:

$$
\begin{aligned}
& \frac{d^{2} x}{d t^{2}}=f\left(s, x(s), \dot{x}(s), \int_{0}^{t} \phi(s, x(s), \dot{x}(s) d s)\right. \\
& \gamma_{1} \dot{x}(0)+\lambda_{1} \dot{x}(T)=\delta \\
& \gamma x(0)+\lambda x(T)=\beta
\end{aligned}
$$

The vector functions

$$
\left.\begin{array}{l}
f(t, x, \dot{x}, y)=\left(f_{1}(t, x, \dot{x}, y), \cdots \cdots, f_{n}(t, x, \dot{x}, y)\right) \\
\phi(t, x, \dot{x})=\left(\phi_{1}(t, x, \dot{x}), \cdots \cdots \cdots, \phi_{n}(t, x, \dot{x})\right)
\end{array}\right\}
$$

are continuous vectors on $t, x, \dot{x}, y$ and defined on the domain

$$
(t, x, \dot{x}, y) \in[0, T] \times D \times D_{1} \times D_{2}
$$

where $D_{1}$ and $D_{2}$ are bounded subset of $R^{n}$,and

$$
\gamma=\left(\gamma_{i j}\right), \lambda=\left(\lambda_{i j}\right), \gamma_{1}=\left(\gamma_{1 i j}\right), \lambda_{1}=\left(\lambda_{1 i j}\right) \text {, }
$$

are positive matrices $(\mathrm{n} \times \mathrm{n}), \beta, \delta \in R^{n}$.

Let the functions $f(t, x, \dot{x}, y)$ and $\phi(t, x, \dot{x})$ satisfy the following inequalities:

$$
\begin{aligned}
& \|f(t, x, \dot{x}, y)\| \leq M,\|\phi(t, x, \dot{x})\| \leq N \\
& \left\|f\left(t, x_{1}, \dot{x}_{1}, y_{1}\right)-f\left(t, x_{2}, \dot{x}_{2}, y_{2}\right)\right\| \leq \mathrm{K}_{1}\left\|\mathrm{x}_{1}-x_{2}\right\|+K_{2}\left\|\dot{x}_{1}-\dot{x}_{2}\right\|+K_{3}\left\|\mathrm{y}_{1}-y_{2}\right\| \\
& \left\|\phi\left(t, x_{1}, \dot{x}_{1}\right)-\phi\left(t, x_{2}, \dot{x}_{2}\right)\right\| \leq L_{1}\left\|x_{1}-x_{2}\right\|+L_{2}\left\|\dot{x}_{1}-\dot{x}_{2}\right\| \\
& \text { for } \quad \text { all }, x, x_{1}, x_{2} \in D, \quad \dot{x}, \dot{x}_{1}, \dot{x}_{2} \in D_{1}, y, y_{1}, y_{2} \in D_{2}, t \in[0, T]
\end{aligned}
$$

$K_{1}, K_{2}, K_{3}, L_{1}, L_{2}, M, N$ are positive constants.

We define the non-empty sets as follows:

$$
\begin{aligned}
& D_{\beta}=D-\left(M \frac{T^{2}}{2}+P_{0}\left(x_{0}\right)\right) ; \\
& D_{1 \beta}=D_{1}-\left(M \frac{T}{2}+P_{1}\left(x_{0}\right)\right) ; \\
& D_{2 \beta}=D_{2}-M_{1} ;
\end{aligned}
$$

and $P_{0}\left(x_{0}\right)=\left(\frac{\mathrm{T}+2 \mathrm{~T}^{2}}{8}\right) M+\left\|x_{0}\right\|+\frac{\mathrm{T}}{4}\|W\|+\|Q\|, P_{1}\left(x_{0}\right)=\frac{2}{\mathrm{~T}}\left\|x_{0}\right\|+2 T M+\frac{2}{\mathrm{~T}}\|Q\|+\|W\|$

$$
\begin{aligned}
& P_{2}\left(x_{0}\right)=2\left\|x_{0}\right\|+T\left\|\dot{x}_{0}\right\|+T^{2} M+2\|Q\|+T\|W\|, W=\lambda_{1}^{-1} \delta-\lambda_{1}^{-1} \gamma_{1} \dot{x}_{0}, Q=\lambda^{-1} \beta-\lambda^{-1} \gamma x_{0}, \\
& p(t)=\left(\frac{t^{2}}{T^{2}}-\frac{2 t}{T}\right), p_{1}(t)=\left(\frac{t^{2}}{T}-t\right), p_{2}(t)=\left(\frac{2 t}{T^{2}}-\frac{2}{T}\right), p_{3}(t)=\left(\frac{2 t}{T^{2}}-1\right), p_{4}(t)=\left(\frac{t^{2}}{T^{2}}-\frac{t}{T}\right)
\end{aligned}
$$

furthermore, we suppose that the largest eigenvalue $\lambda$ of the following matrix. 


$$
\Lambda_{0}=\left(\begin{array}{ll}
{\left[M \frac{T^{2}}{2}+\frac{3 T^{2}}{8}\right]\left(K_{1}+K_{3} L_{1} T\right)} & {\left[M \frac{T^{2}}{2}+\frac{3 T^{2}}{8}\right]\left(K_{2}+K_{3} L_{2} T\right)} \\
{\left[\frac{T}{2}+2 T\right]\left(K_{1}+K_{3} L_{1} T\right)} & {\left[\frac{T}{2}+2 T\right]\left(K_{2}+K_{3} L_{2} T\right)}
\end{array}\right)
$$

is less than one.i.e.

$$
\lambda_{1}=0, \lambda_{2}=\left[\left[M \frac{T^{2}}{2}+\frac{3 T^{2}}{8}\right]\left(K_{1}+K_{3} L_{1} T\right)+\left[\frac{T}{2}+2 T\right]\left(K_{2}+K_{3} L_{2} T\right)\right] \leq 1
$$

\section{LEMMA 1 [6]}

Let $\mathrm{f}(\mathrm{t})$ be a continuous vector function in the interval $0 \leq t \leq T$, then $\left\|\int_{0}^{t}\left(f(s)-\frac{1}{T} \int_{0}^{T} f(s) d s\right) d s\right\| \leq M \alpha(t)$

where

$$
\alpha(t)=2 t\left(1-\frac{t}{T}\right), \quad M=\max _{t \in[0, T]}\|f(t)\| .
$$

\section{LEMMA 2}

Let $\mathrm{f}(\mathrm{t})$ be a continuous vector function in the interval $0 \leq t \leq T$, then

$\left\|\int_{0}^{t}\left[\left(\int_{0}^{t} f(s) d s\right)-\frac{1}{T} \int_{0}^{T}\left(\int_{0}^{t} f(s) d s\right) d s\right] d s\right\| \leq T M \alpha(t) \leq M \frac{T^{2}}{2}$

Where $\left\|\int_{0}^{t} f(s) d s\right\| \leq \int_{0}^{t}\|f(s)\| d s \leq t M \leq T M$

\section{PROOF:}

From the following inequality

$$
\begin{aligned}
\left.\| \int_{0}^{t}\left[\left(\int_{0}^{s} f(s) d s\right)-\frac{1}{T} \int_{0}^{T} \int_{0}^{s} f(s) d s\right) d s\right] d s \| & \leq \int_{0}^{t}\left(\int_{0}^{s} f(s) d s\right) d s-\frac{t}{T} \int_{0}^{t}\left(\int_{0}^{s} f(s) d s\right) d s-\frac{t}{T} \int_{t}^{T}\left(\int_{0}^{s} f(s) d s\right) d s \| \\
& \leq\left\|\left(1-\frac{t}{T}\right) \int_{0}^{t}\left(\int_{0}^{s} f(s) d s\right) d s-\frac{t}{T} \int_{t}^{T}\left(\int_{0}^{s} f(s) d s\right) d s\right\| \\
& \leq\left(1-\frac{t}{T}\right) \int_{0}^{t}\left\|\int_{0}^{s} f(s) d s\right\| d s+\frac{t^{T}}{T} \int_{t}^{t}\left\|\int_{0}^{s} f(s) d s\right\| d s \\
& \leq\left(1-\frac{t}{T}\right) \int_{0}^{t} T M d s+\frac{t}{T} \int_{t}^{T} T M d s \\
& \leq T M \alpha(t) \leq M \frac{T^{2}}{2}
\end{aligned}
$$

We define the operator $\mathrm{L}$ as:

$$
L\left(\int_{0}^{t} f(s) d s\right)=\int_{0}^{t}\left[\left(\int_{0}^{s} f(s) d s\right)-\frac{1}{T} \int_{0}^{T}\left(\int_{0}^{s} f(s) d s\right) d s\right] d s
$$


Since $\mathrm{f}(\mathrm{t})$ is continuous function on the interval $[0, T]$. then the integral $\left(\int_{0}^{t} f(s) d s\right)$ is continuous on the same interval and $L\left(\int_{0}^{t} f(s) d s\right)$ is also continuous on the same interval .

By lemma 2 .we get $\left\|L\left(\int_{0}^{t} f(s) d s\right)\right\| \leq \alpha(t) T M \leq M \frac{T^{2}}{2} \quad$ For all $\quad t \in[0, T], \alpha(t) \leq \frac{T}{2}$.

\section{Approximate solution}

The investigation of approximate solution of the problem (A) will be introduced by the following theorem.

\section{THEOREM 1}

If the system (A) satisfies the inequalities (1.8), (1.9), (1.10) and then the sequence of functions defined by:

$$
\begin{aligned}
& x_{m}\left(t, x_{0}\right)=x_{0}+L\left(\int_{0}^{t} f\left(s, x_{m-1}\left(s, x_{0}\right), \dot{x}_{m-1}\left(s, x_{0}\right), \int_{0}^{s} \phi\left(s, x_{m-1}\left(s, x_{0}\right), \dot{x}_{m-1}\left(s, x_{0}\right) d s\right) d s\right)\right. \\
& +p_{4}(t) \int_{0}^{T} \int_{0}^{t} f\left(s, x_{m-1}\left(s, x_{0}\right), \dot{x}_{m-1}\left(s, x_{0}\right), \int_{0}^{s} \phi\left(s, x_{m-1}\left(s, x_{0}\right), \dot{x}_{m-1}\left(s, x_{0}\right) d s\right) d s d t-\right. \\
& -p_{1}(t) \int_{0}^{T} f\left(s, x_{m-1}\left(s, x_{0}\right), \dot{x}_{m-1}\left(s, x_{0}\right), \int_{0}^{s} \phi\left(s, x_{m-1}\left(s, x_{0}\right), \dot{x}_{m-1}\left(s, x_{0}\right) d s\right) d s+p(t) x_{0}+P_{1}(t) W+P(t) Q\right. \\
& x_{0}\left(t, x_{0}\right)=x_{0}, \frac{d x_{m}\left(t, x_{0}\right)}{d t}=\dot{x}_{m}\left(t, x_{0}\right)
\end{aligned}
$$

As $m \rightarrow \infty$

converges uniformly in the domain:

$$
\left(t, x_{0}\right) \in[0, T] \times D_{\beta}
$$

for $x_{0} \in D_{\beta}$,

to the function $x_{\infty}\left(t, x_{0}\right)$ which is satisfying the integral equation

$$
\begin{aligned}
& x\left(t, x_{0}\right)=x_{0}+L\left(\int_{0}^{t} f\left(s, x\left(s, x_{0}\right), \dot{x}\left(s, x_{0}\right), \int_{0}^{s} \phi\left(s, x\left(s, x_{0}\right), \dot{x}\left(s, x_{0}\right) d s\right) d s\right)\right. \\
& +p_{4}(t) \int_{0}^{T} \int_{0}^{t} f\left(s, x\left(s, x_{0}\right), \dot{x}\left(s, x_{0}\right), \int_{0}^{s} \phi\left(s, x\left(s, x_{0}\right), \dot{x}\left(s, x_{0}\right) d s\right) d s d t-\quad \ldots(2.15)\right. \\
& -p_{1}(t) \int_{0}^{T} f\left(s, x\left(s, x_{0}\right), \dot{x}\left(s, x_{0}\right), \int_{0}^{s} \phi\left(s, x\left(s, x_{0}\right), \dot{x}\left(s, x_{0}\right) d s\right) d s+p(t) x_{0}+P_{1}(t) W+P(t) Q\right.
\end{aligned}
$$

And has a unique solution provided that

$$
\left\|x_{\infty}\left(t, x_{0}\right)-x_{0}\right\| \leq M \frac{T^{2}}{2}+P_{0}\left(x_{0}\right)
$$

\section{PROOF:}

Setting m=1and using lemma 2 in (2.13), we have 
$\left\|x_{1}\left(t, x_{0}\right)-x_{0}\right\| \leq \| x_{0}+L\left(\int_{0}^{t} f\left(s, x_{0}\left(s, x_{0}\right), \dot{x}_{0}\left(s, x_{0}\right), \int_{0}^{s} \phi\left(t, x_{0}\left(t, x_{0}\right), \dot{x}_{0}\left(t, x_{0}\right) d t\right) d s\right)\right.$

$+p_{4}(t) \int_{0}^{T} \int_{0}^{t} f\left(s, x_{0}\left(s, x_{0}\right), \dot{x}_{0}\left(s, x_{0}\right), \int_{0}^{s} \phi\left(s, x_{0}\left(s, x_{0}\right), \dot{x}_{0}\left(s, x_{0}\right) d s\right) d s d t-\right.$

$-p_{1}(t) \int_{0}^{T} f\left(s, x_{0}\left(s, x_{0}\right), \dot{x}_{0}\left(s, x_{0}\right), \int_{0}^{s} \phi\left(s, x_{0}\left(s, x_{0}\right), \dot{x}_{0}\left(s, x_{0}\right) d s\right) d s+p(t) x_{0}+P_{1}(t) W+P(t) Q-x_{0} \|\right.$

$\left\|x_{1}\left(t, x_{0}\right)-x_{0}\right\| \leq M T \alpha(t)+P_{0}\left(x_{0}\right) \leq M \frac{T^{2}}{2}+P_{0}\left(x_{0}\right)$

$x_{1}\left(t, x_{0}\right) \in D$ for all,$x_{0} \in D_{\beta}$

on differentiating $x\left(t, x_{0}\right)$ from (2.15) and taking the norm, we have $\left\|\dot{x}\left(t, x_{0}\right)\right\|=\| p_{2}(t) x_{0}+\int_{0}^{t}\left(f\left(s, x\left(s, x_{0}\right), \dot{x}\left(s, x_{0}\right), \int_{0}^{s} \phi\left(s, x\left(s, x_{0}\right), \dot{x}\left(s, x_{0}\right) d s\right)-\right.\right.$ $-\frac{1}{T} \int_{0}^{T} f\left(s, x\left(s, x_{0}\right), \dot{x}\left(s, x_{0}\right), \int_{0}^{s} \phi\left(s, x\left(s, x_{0}\right), \dot{x}\left(s, x_{0}\right) d s\right) d s\right) d s+$

$+p_{2}(t) \int_{0}^{T} \int_{0}^{t} f\left(s, x\left(s, x_{0}\right), \dot{x}\left(s, x_{0}\right), \int_{0}^{s} \phi\left(s, s, x\left(s, x_{0}\right), \dot{x}\left(s, x_{0}\right) d s\right) d s d t+\right.$

$+\left(1-\frac{t}{T}\right) \int_{0}^{T} f\left(s, x\left(s, x_{0}\right) \dot{x}\left(s, x_{0}\right), \int_{0}^{s} \phi\left(s, x\left(s, x_{0}\right), \dot{x}\left(s, x_{0}\right) d s\right) d s\right.$

$-p_{2}(t) Q+p_{3}(t) W \|$

Or

$$
\begin{gathered}
\left\|\dot{x}\left(t, x_{0}\right)\right\| \leq \frac{2}{T}\left\|x_{0}\right\|+M \alpha(t)+2 T M+\frac{2}{T}\|Q\|+\|W\| \\
\leq M \frac{T}{2}+P_{1}\left(x_{0}\right)
\end{gathered}
$$

From (2.18),(2.21) we get

$$
\begin{aligned}
\left\|y_{1}\left(t, x_{0}\right)-y_{0}(t)\right\| \leq \| \int_{0}^{t} \phi(s, & \left.x_{1}\left(s, x_{0}\right), \dot{x}_{1}\left(s, x_{0}\right)\right) d t-\phi\left(s, x_{0}\left(s, x_{0}\right), 0\right) d t \| \\
& \leq \int_{0}^{t}\left\|\phi\left(s, x_{1}\left(s, x_{0}\right), \dot{x}_{1}\left(s, x_{0}\right)\right) d t-\phi\left(s, x_{0}\left(s, x_{0}\right), 0\right)\right\| d t \\
& \leq T\left(L_{1}\left\|x_{1}\left(t, x_{0}\right)-x_{0}\right\|+L_{2}\left\|\dot{x}_{1}\left(t, x_{0}\right)\right\|\right) \\
& \leq T\left(L_{1} M \frac{T^{2}}{2}+P_{0}\left(x_{0}\right)+L_{2} M \frac{T^{2}}{2}+P_{1}\left(x_{0}\right)\right) \\
& \leq M_{1}
\end{aligned}
$$


where $y_{0}(t)=\int_{0}^{t} \phi\left(s, x_{0}\left(s, x_{0}\right), 0\right) d s \quad, \quad x_{0} \in D_{M}$

By lemma 2 and the sequence of the functions (2.14) when $m=1$ we get $x_{1}\left(t, x_{0}\right) \in D$ for all $t \in[0, T]$ and for all $x_{0} \in D_{\beta}$

by induction we have:

$\left\|x_{m}\left(t, x_{0}\right)-x_{0}\right\| \leq M \frac{T^{2}}{2}+P_{0}\left(x_{0}\right)$
$\left\|y_{m}\left(t, x_{0}\right)-y_{0}(t)\right\| \leq M_{1}$
$x_{m}\left(t, x_{0}\right) \in D$ for all,$x_{0} \in D_{\beta}$

and $y_{m}\left(t, x_{0}\right) \in D_{2}$ for all , $y_{0} \in D_{\beta 2}$

and $y_{m}(t)=\int_{0}^{t} \phi\left(s, x_{m}\left(s, x_{0}\right), \dot{x}_{m}\left(s, x_{0}\right)\right) d s \quad, \quad \mathrm{~m}=0,1,2,3 \ldots \ldots$

Now we prove that the sequence of functions $\left\{x_{m}\left(t, x_{0}\right)\right\}_{m=0}^{\infty}$ converges uniformly in the domain (1.7) ,by (2.13) and lemma 2, we have $\left\|x_{m+1}\left(t, x_{0}\right)-x_{m}\left(t, x_{0}\right)\right\| \leq T \alpha(t)\left[\left(K_{1}+K_{3} L_{1} T\right)\left\|x_{m+1}\left(t, x_{0}\right)-x_{m}\left(t, x_{0}\right)\right\|\right.$

$\left.+\left(K_{2}+K_{3} L_{2} T\right)\left\|\dot{x}_{m+1}\left(t, x_{0}\right)-\dot{x}_{m}\left(t, x_{0}\right)\right\|\right]+\frac{T^{2}}{8}\left[\left(K_{1}+K_{3} L_{1} T\right)\left\|x_{m+1}\left(t, x_{0}\right)-x_{m}\left(t, x_{0}\right)\right\|\right.$

$\left.+\left(K_{2}+K_{3} L_{2} T\right)\left\|\dot{x}_{m+1}\left(t, x_{0}\right)-\dot{x}_{m}\left(t, x_{0}\right)\right\|\right]+\frac{T^{2}}{4}\left[\left(K_{1}+K_{3} L T\right)\left\|x_{m+1}\left(t, x_{0}\right)-x_{m}\left(t, x_{0}\right)\right\|\right.$

$+\left(K_{2}+K_{3} L_{2} T\right)\left\|\dot{x}_{m+1}\left(t, x_{0}\right)-\dot{x}_{m}\left(t, x_{0}\right)\right\|$.

$$
\begin{aligned}
& \leq\left[\frac{T^{2}}{2}+\frac{3 T^{2}}{8}\right]\left[\left(K_{1}+K_{3} L_{1} T\right)\left\|x_{m+1}\left(t, x_{0}\right)-x_{m}\left(t, x_{0}\right)\right\|+\right. \\
& \left.+\left(K_{2}+K_{3} L_{2} T\right)\left\|\dot{x}_{m+1}\left(t, x_{0}\right)-\dot{x}_{m}\left(t, x_{0}\right)\right\|\right]
\end{aligned}
$$

By (1.8), (1.9), (1.10) and lemma 1 , we have

$$
\begin{aligned}
\left\|\dot{x}_{m+1}\left(t, x_{0}\right)-\dot{x}_{m}\left(t, x_{0}\right)\right\| \leq \alpha(t)\left[\left(K_{1}+K_{3} L_{1} T\right)\left\|x_{m+1}\left(t, x_{0}\right)-x_{m}\left(t, x_{0}\right)\right\|\right. \\
\left.+\left(K_{2}+K_{3} L_{2} T\right)\left\|\dot{x}_{m+1}\left(t, x_{0}\right)-\dot{x}_{m}\left(t, x_{0}\right)\right\|\right]+2 T\left[\left(K_{1}+K_{3} L T\right)\left\|x_{m+1}\left(t, x_{0}\right)-x_{m}\left(t, x_{0}\right)\right\|\right. \\
\leq \\
\leq[\alpha(t)+2 T]\left[\left[\left(K_{1}+K_{3} L_{1} T\right)\left\|x_{m+1}\left(t, x_{0}\right)-x_{m}\left(t, x_{0}\right)\right\|+\right.\right. \\
\left.+\left(K_{2}+K_{3} L_{2} T\right)\left\|\dot{x}_{m+1}\left(t, x_{0}\right)-\dot{x}_{m}\left(t, x_{0}\right)\right\|\right] \\
\leq\left[\frac{T}{2}+2 T\right]\left[\left[\left(K_{1}+K_{3} L_{1} T\right)\left\|x_{m+1}\left(t, x_{0}\right)-x_{m}\left(t, x_{0}\right)\right\|+\right.\right. \\
\left.+\left(K_{2}+K_{3} L_{2} T\right)\left\|\dot{x}_{m+1}\left(t, x_{0}\right)-\dot{x}_{m}\left(t, x_{0}\right)\right\|\right]
\end{aligned}
$$

Rewrite the inequalities (2.25), (2.26) in vector form as

$$
V_{m+1}(t) \leq \Lambda(t) V_{m}(t)
$$

where

$$
V_{m+1}(t)=\left(\begin{array}{l}
\left\|x_{m+1}\left(t, x_{0}\right)-x_{m}\left(t, x_{0}\right)\right\| \\
\left\|\dot{x}_{m+1}\left(t, x_{0}\right)-\dot{x}_{m}\left(t, x_{0}\right)\right\|
\end{array}\right) ;
$$




$$
\Lambda(t)=\left(\begin{array}{lc}
{\left[T o(t)+\frac{3 T^{2}}{8}\right]\left(K_{1}+K_{3} L_{1} T\right)} & {\left[T o(t)+\frac{3 T^{2}}{8}\right]\left(K_{2}+K_{3} L_{2} T\right)} \\
{[\alpha(t)+2 T]\left(K_{1}+K_{3} L_{1} T\right)} & {[\alpha(t)+2 T]\left(K_{2}+K_{3} L_{2} T\right)}
\end{array}\right)
$$

it follows from inequality (2.27) that

$$
V_{m+1} \leq \Lambda_{0} V_{m}
$$

where

$$
\Lambda_{0}=\max _{t \in[0, T]}|\Lambda(t)|
$$

From (2.31) and by iteration, we have

$$
V_{m+1} \leq \Lambda_{0}^{m} V_{1}
$$

which leads to the estimation

$$
\sum_{i=1}^{m} V_{i} \leq \sum_{i=1}^{m} \Lambda_{0}^{i-1} V_{1}
$$

since the matrix $\Lambda_{0}$ has eigenvalues (1.12).

and thus the series (2.34) is uniformly convergent, i.e.

$$
\lim _{m \rightarrow \infty} \sum_{i=1}^{m} \Lambda_{0}^{i-1} V_{1}=\sum_{i=1}^{\infty} \Lambda_{0}^{i-1} V_{1}=\left(E-\Lambda_{0}\right)^{-1} V_{1}
$$

Where $\mathrm{E}$ is the identity matrix.

The limiting relation (2.34) implies to the uniformly convergent of the sequences $\left[x_{m}\left(t, x_{0}\right)\right],\left[\dot{x}_{m}\left(t, x_{0}\right)\right]$ in the domain (2.14)

Let:

$$
\left.\begin{array}{l}
\lim _{m \rightarrow \infty} x_{m}\left(t, x_{0}\right)=x_{\infty}\left(t, x_{0}\right) \\
\lim _{m \rightarrow \infty} \dot{x}_{m}\left(t, x_{0}\right)=\dot{x}_{\infty}\left(t, x_{0}\right)
\end{array}\right\}
$$

by inequality (2.33), the estimation

$$
\left(\begin{array}{l}
\left\|x_{\infty}\left(t, x_{0}\right)-x_{m}\left(t, x_{0}\right)\right\| \\
\left\|\dot{x}_{\infty}\left(t, x_{0}\right)-\dot{x}_{m}\left(t, x_{0}\right)\right\|
\end{array}\right) \leq \Lambda_{0}^{m}\left(E-\Lambda_{0}\right)^{-1} V_{1}
$$

is true for $\mathrm{m}=1,2,3, \ldots$

Thus $x_{\infty}\left(t, x_{0}\right)$ is solutions of the integral equations (2.15).

Finally, we have to show that $x\left(t, x_{0}\right)$ is a unique solution of (A), on the contrary we suppose that there are at least two different solutions $x\left(t, x_{0}\right), z\left(t, x_{0}\right)$ of (A).

From (1.15),(1.16) the following inequalities hold:

$$
\begin{aligned}
& \left\|x\left(t, x_{0}\right)-z\left(t, x_{0}\right)\right\| \leq\left[\frac{T^{2}}{2}+\frac{3 T^{2}}{8}\right]\left[\left(K_{1}+K_{3} L_{1} T\right)\left\|x\left(t, x_{0}\right)-z\left(t, x_{0}\right)\right\|+\right. \\
& \left.+\left(K_{2}+K_{3} L_{2} T\right)\left\|\dot{x}\left(t, x_{0}\right)-\dot{z}\left(t, x_{0}\right)\right\|\right]
\end{aligned}
$$


$\left\|\dot{x}\left(t, x_{0}\right)-\dot{z}\left(t, x_{0}\right)\right\| \leq\left[\frac{T}{2}+2 T\right]\left[\left(K_{1}+K_{3} L_{1} T\right)\left\|x\left(t, x_{0}\right)-z\left(t, x_{0}\right)\right\|+\right.$

$\left.+\left(K_{2}+K_{3} L_{2} T\right)\left\|\dot{x}\left(t, x_{0}\right)-\dot{z}\left(t, x_{0}\right)\right\|\right]$

thus:

$\left(\begin{array}{l}\left\|x\left(t, x_{0}\right)-z\left(t, x_{0}\right)\right\| \\ \left\|\dot{x}\left(t, x_{0}\right)-\dot{z}\left(t, x_{0}\right)\right\|\end{array}\right) \leq \Lambda_{0}\left(\begin{array}{l}\left\|x\left(t, x_{0}\right)-z\left(t, x_{0}\right)\right\| \\ \left\|\dot{x}\left(t, x_{0}\right)-\dot{z}\left(t, x_{0}\right)\right\|\end{array}\right)$

by iteration we find that

$\left(\begin{array}{l}\left\|x\left(t, x_{0}\right)-z\left(t, x_{0}\right)\right\| \\ \left\|\dot{x}\left(t, x_{0}\right)-\dot{z}\left(t, x_{0}\right)\right\|\end{array}\right) \leq \Lambda_{0}^{m}\left(\begin{array}{l}\left\|x\left(t, x_{0}\right)-z\left(t, x_{0}\right)\right\| \\ \left\|\dot{x}\left(t, x_{0}\right)-\dot{z}\left(t, x_{0}\right)\right\|\end{array}\right)$

Now from the condition (1.12) we have $\Lambda^{m} \rightarrow 0$ as $m \rightarrow \infty$, and hence the limit of the last inequality gives $x\left(t, x_{0}\right)=z\left(t, x_{0}\right), \dot{x}\left(t, x_{0}\right)=\dot{z}\left(t, x_{0}\right)$ which prove that the solution is unique and this completes the proof of theorem 1. .

\section{Existence of solution}

The problem of existence solution of (A) is uniquely connected with the existence of zeros of the functions, $\Delta\left(0, x_{0}\right)$ which has the form.

$$
\begin{aligned}
\Delta\left(0, x_{0}\right)=2 x_{0}+ & \dot{x}_{0}-\int_{0}^{T} f\left(s, x_{\infty}\left(s, x_{0}\right), \dot{x}_{\infty}\left(s, x_{0}\right), \int_{0}^{s} \phi\left(s, x_{\infty}\left(s, x_{0}\right), \dot{x}_{\infty}\left(s, x_{0}\right) d s\right) d s-\right. \\
& -\frac{2}{T} \int_{0}^{T} \int_{0}^{t} f\left(s, x_{\infty}\left(s, x_{0}\right), \dot{x}_{\infty}\left(s, x_{0}\right), \int_{0}^{s} \phi\left(s, x_{\infty}\left(s, x_{0}\right), \dot{x}_{\infty}\left(s, x_{0}\right) d s\right)\right) d s d t W-\frac{2}{T} Q,
\end{aligned}
$$

since this functions are approximately determined from the sequence of functions:

$$
\begin{aligned}
\Delta_{m}\left(0, x_{0}\right)=2 x_{0} & +\dot{x}_{0}-\int_{0}^{T} f\left(s, x_{m}\left(s, x_{0}\right), \dot{x}_{m}\left(s, x_{0}\right), \int_{0}^{s} \phi\left(s, x_{m}\left(s, x_{0}\right), \dot{x}_{m}\left(s, x_{0}\right) d s\right)\right) d s- \\
& -\frac{2}{T} \int_{0}^{T} \int_{0}^{t} f\left(s, x_{m}\left(s, x_{0}\right), \dot{x}_{m}\left(s, x_{0}\right), \int_{0}^{s} \phi\left(s, x_{m}\left(s, x_{0}\right), \dot{x}_{m}\left(s, x_{0}\right) d s\right)\right) d s d t W-\stackrel{2}{T} Q
\end{aligned}
$$

for $\mathrm{m}=0,1,2,3 \ldots$

\section{LEMMA 3}

Suppose that the conditions of theorem 1 are satisfied, then the following inequality

$$
\left\|\Delta\left(0, x_{0}\right)-\Delta_{m}\left(0, x_{0}\right)\right\| \leq\left\langle\left(\begin{array}{c}
2 T\left(K_{1}+K_{3} L_{1} T\right) \\
2 T\left(K_{2}+K_{3} L_{2} T\right)
\end{array}\right), \Lambda_{0}^{m}\left(E-\Lambda_{0}\right)^{-1} V_{1}\right\rangle=\sigma_{m}
$$

Satisfied for $m \geq 0, y_{0} \in D_{\beta 2}, x_{0} \in D_{\beta}$.

\section{PROOF:}

From (3.40) and (3.41), we get 


$$
\begin{aligned}
\left\|\Delta\left(0, x_{0}\right)-\Delta_{m}\left(0, x_{0}\right)\right\| & \leq 2 T\left(K_{1}+K_{3} L_{1} T\right)\left\|x_{\infty}\left(t, x_{0}\right)-x_{m}\left(t, x_{0}\right)\right\| \\
+2 T( & \left.K_{2}+K_{3} L_{2} T\right)\left\|\dot{x}_{\infty}\left(t, x_{0}\right)-\dot{x}_{m}\left(t, x_{0}\right)\right\| \\
& \leq\left\langle\left(\begin{array}{c}
2 T\left(K_{1}+K_{3} L_{1} T\right) \\
2 T\left(K_{2}+K_{3} L_{2} T\right)
\end{array}\right), \Lambda_{0}^{m}\left(E-\Lambda_{0}\right)^{-1} V_{1}\right\rangle=\sigma_{m}
\end{aligned}
$$

Now we prove the following theorem taking into account that the inequality (3.42) will be satisfied for all $m \geq 0$.

\section{THEOREM 2}

Suppose that for $m \geq 0$ the sequence of functions $\Delta\left(0, x_{0}\right)$ which are defined in (3.41) satisfies the inequality.

$$
\begin{aligned}
& \min _{a+h \leq x_{0} \leq b-h} \Delta_{m}\left(0, x_{0}\right) \leq-\sigma_{m} \\
& \operatorname{maX}_{a+h \leq x_{0} \leq b-h} \Delta_{m}\left(0, x_{0}\right) \geq \sigma_{m} \\
& \text { Where, } h=M \frac{T^{2}}{2}+P_{0}\left(x_{0}\right),
\end{aligned}
$$

then the boundary value problem (A) has solutions $x=x\left(t, x_{0}\right)$ such that $a+h \leq x_{0} \leq b-h$.

\section{PROOF:}

Let $x_{1}, x_{2}$ be any point in the interval $[a+h, b-h]$ such that

$$
\begin{aligned}
& \Delta\left(0, x_{1}\right)=\min _{a+h \leq x_{0} \leq b-h} \Delta\left(0, x_{0}\right) \\
& a+h \leq x_{0} \leq b-h \\
& \Delta\left(0, x_{2}\right)=\max _{a+h \leq x_{0} \leq b-h} \Delta\left(0, x_{0}\right)
\end{aligned}
$$

by using the inequalities (3.42),(3.44) we have:

$$
\begin{aligned}
& \Delta\left(0, x_{1}\right)=\Delta_{m}\left(0, x_{1}\right)+\left[\Delta\left(0, x_{1}\right)-\Delta_{m}\left(0, x_{1}\right)\right] \leq 0 \\
& \Delta\left(0, x_{2}\right)=\Delta_{m}\left(0, x_{2}\right)+\left[\Delta\left(0, x_{2}\right)-\Delta_{m}\left(0, x_{2}\right)\right] \geq 0
\end{aligned}
$$

From the continuity of (3.40) and from (3.46) there exists a singular point $x_{\infty}=x_{0}, x_{\infty} \in\left[x_{1}, x_{2}\right\rfloor$, such that: $\Delta\left(0, x_{\infty}\right)=0$

thus $x=x\left(t, x_{0}\right)$ is a solution of $(\mathrm{A})$.

\section{REMARK}

When $R^{n}=R^{1}$ i.e. $X_{0}$ is a scalar, theorem 2 can be strengthen by omitting the requirement that the singular point should be isolated. (For this remark see [5])

\section{THEOREM 3}

If the function $\Delta\left(0, x_{0}\right)$ is defined by

$$
\Delta: D_{\beta} \rightarrow R^{n}
$$




$$
\begin{aligned}
\Delta\left(0, x_{0}\right) & =2 x_{0}+\dot{x}_{0}-\int_{0}^{T} f\left(s, x_{\infty}\left(s, x_{0}\right) \dot{x}_{\infty}\left(s, x_{0}\right), y_{\infty}\left(s, x_{0}\right)\right) d s- \\
& -\frac{2}{T} \int_{0}^{T} \int_{0}^{t} f\left(s, x_{\infty}\left(s, x_{0}\right), \dot{x}_{\infty}\left(s, x_{0}\right), y_{\infty}\left(s, x_{0}\right)\right) d s d t\left(\lambda_{1}^{-1} \delta-\lambda_{1}^{-1} \gamma_{1}\right) \dot{x}_{0} \frac{2}{T}\left(\lambda^{-1} \beta-\lambda^{-1} \gamma\right) x_{0} .
\end{aligned}
$$

Where the function $x_{\infty}\left(t, x_{0}\right)$ is limit of function (2.13) then the inequalities

$$
\left\|\Delta\left(0, x_{0}\right)\right\| \leq M T+\frac{P_{2}\left(x_{0}\right)}{T}
$$

and

$$
\begin{aligned}
\left\|\Delta\left(0, x_{0}^{1}\right)-\Delta\left(0, x_{0}^{2}\right)\right\| \leq\left[E_{7}+2 T E_{1} E_{11}\left(E_{9} E_{3} E_{2} E_{10} E_{7}+E_{9} E_{5}\right)+\right. \\
\left.+2 T E_{2} E_{12}\left(E_{10} E_{4} E_{1} E_{9} E_{5}+E_{10} E_{9}\right)\right]\left\|x_{0}^{1}-x_{0}^{2}\right\| \\
\quad+\left[E_{5}+2 T E_{1} E_{11}\left(E_{9} E_{3} E_{2} E_{10} E_{8}+E_{9} E_{5}\right)+\right. \\
\left.+2 T E_{2} E_{12}\left(E_{10} E_{4} E_{1} E_{9} E_{6}+E_{10} E_{8}\right)\right]\left\|\dot{x}_{0}^{1}-\dot{x}_{0}^{2}\right\|
\end{aligned}
$$

Are satisfies for $x_{0}, x_{0}^{1}, x_{0}^{2} \in D_{\beta}$

Where, $E_{1}=\left(K_{1}+K_{3} L_{1} T\right), E_{2}=\left(K_{2}+K_{3} L_{2} T\right), E_{3}=\left(\frac{T^{2}}{2}+\frac{3 T^{2}}{8}\right), E_{4}=\left(\frac{T}{2}+2 T\right)$

$$
\begin{aligned}
& E_{5}=\left(1+\left\|\lambda^{-1} \beta\right\|\right), E_{6}=\left(\frac{T}{4}\left\|\lambda_{1}^{-1} \delta\right\|\right), E_{7}=\left(\frac{2}{T}+\frac{2}{T}\left\|\lambda^{-1} \beta\right\|\right), E_{8}=\left(\left\|\lambda_{1}^{-1} \delta\right\|\right), \\
& E_{9}=\left(1-E_{3} E_{1}\right)^{-1}, E_{10}=\left(1-E_{4} E_{2}\right)^{-1}, E_{11}=\left(1-E_{9} E_{3} E_{2} E_{10} E_{4} E_{1}\right)^{-1}, \\
& E_{12}=\left(1-E_{10} E_{4} E_{1} E_{9} E_{3} E_{2}\right)^{-1}
\end{aligned}
$$

\section{PROOF:}

From $x_{\infty}\left(t, x_{0}\right)$ the function $\Delta\left(0, x_{0}\right)$ that continuous and bounded by

$$
\begin{aligned}
& M+\frac{B_{1}}{T} \quad x_{0} \in D_{\beta}, \dot{x}_{0} \in D_{\beta 1} \text { and } \\
& \begin{aligned}
\left\|\Delta\left(0, x_{0}^{1}\right)-\Delta\left(0, x_{0}^{2}\right)\right\| \leq \mathrm{E}_{7} \| & x_{0}^{1}-x_{0}^{2}\left\|+E_{5}\right\| \dot{x}_{0}^{1}-\dot{x}_{0}^{2}\left\|+2 T E_{1}\right\| x_{\infty}\left(t, x_{0}^{1}\right)-x_{\infty}\left(t, x_{0}^{2}\right) \| \\
& +2 \mathrm{TE}_{2}\left\|\dot{x}_{\infty}\left(t, x_{0}^{1}\right)-\dot{x}_{\infty}\left(t, x_{0}^{2}\right)\right\| \ldots(3.50)
\end{aligned}
\end{aligned}
$$

and the functions $x_{\infty}\left(t, x_{0}^{1}\right), x_{\infty}\left(t, x_{0}^{2}\right)$ are solution of integral equations:

$$
\begin{aligned}
& x\left(t, x_{0}^{k}\right)=x_{0}+L\left(\int_{0}^{t} f\left(s, x\left(t, x_{0}^{k}\right), \dot{x}\left(t, x_{0}^{k}\right), \int_{0}^{s} \phi\left(s, x\left(s, x_{0}^{k}\right), \dot{x}\left(s, x_{0}^{k}\right) d s\right)\right) d s\right) \\
& +p_{4}(t) \int_{0}^{T} \int_{0}^{t} f\left(s, x\left(t, x_{0}^{k}\right), \dot{x}\left(t, x_{0}^{k}\right), \int_{0}^{s} \phi\left(s, s, x\left(s, x_{0}^{k}\right), \dot{x}\left(s, x_{0}^{k}\right) d s\right) d s d t-\right. \\
& -p_{1}(t) \int_{0}^{T} f\left(s, x\left(s, x_{0}^{k}\right), \dot{x}\left(s, x_{0}^{k}\right), \int_{0}^{s} \phi\left(s, x\left(s, x_{0}^{k}\right), \dot{x}\left(s, x_{0}^{k}\right) d s\right) d s+\right. \\
& +p(t) x_{0}+P_{1}(t) \lambda_{1}^{-1} \delta-\lambda_{1}^{-1} \gamma_{1} \dot{x}_{0}+P(t) \lambda^{-1} \beta-\lambda^{-1} \gamma x_{0}
\end{aligned}
$$


By (3.51) and lemma 2 we get

$$
\begin{aligned}
\left\|x_{\infty}\left(t, x_{0}^{1}\right)-x_{\infty}\left(t, x_{0}^{2}\right)\right\| & \leq E_{3} E_{1}\left\|x_{\infty}\left(t, x_{0}^{1}\right)-x_{\infty}\left(t, x_{0}^{2}\right)\right\|+ \\
+ & E_{3} E_{2}\left\|\dot{x}_{\infty}\left(t, x_{0}^{1}\right)-\dot{x}_{\infty}\left(t, x_{0}^{2}\right)\right\|+E_{5}\left\|x_{0}^{1}-x_{0}^{2}\right\|+E_{6}\left\|\dot{x}_{0}^{1}-\dot{x}_{0}^{2}\right\| \ldots \\
\left\|\dot{x}_{\infty}\left(t, x_{0}^{1}\right)-\dot{x}_{\infty}\left(t, x_{0}^{2}\right)\right\| & \leq E_{4} E_{1}\left\|x_{\infty}\left(t, x_{0}^{1}\right)-x_{\infty}\left(t, x_{0}^{2}\right)\right\|+ \\
+ & E_{4} E_{2}\left\|\dot{x}_{\infty}\left(t, x_{0}^{1}\right)-\dot{x}_{\infty}\left(t, x_{0}^{2}\right)\right\|+E_{7}\left\|x_{0}^{1}-x_{0}^{2}\right\|+E_{8}\left\|\dot{x}_{0}^{1}-\dot{x}_{0}^{2}\right\| \ldots(3.5)
\end{aligned}
$$

then

$$
\begin{aligned}
& \left\|x_{\infty}\left(t, x_{0}^{1}\right)-x_{\infty}\left(t, x_{0}^{2}\right)\right\| \leq E_{9} E_{3} E_{2}\left\|\dot{x}_{\infty}\left(t, x_{0}^{1}\right)-\dot{x}_{\infty}\left(t, x_{0}^{2}\right)\right\|+ \\
& +E_{9} E_{5}\left\|x_{0}^{1}-x_{0}^{2}\right\|+E_{9} E_{6}\left\|\dot{x}_{0}^{1}-\dot{x}_{0}^{2}\right\| \\
& \left\|\dot{x}_{\infty}\left(t, x_{0}^{1}\right)-\dot{x}_{\infty}\left(t, x_{0}^{2}\right)\right\| \leq E_{10} E_{4} E_{1}\left\|x_{\infty}\left(t, x_{0}^{1}\right)-x_{\infty}\left(t, x_{0}^{2}\right)\right\|+ \\
& +E_{10} E_{7}\left\|x_{0}^{1}-x_{0}^{2}\right\|+E_{10} E_{8}\left\|\dot{x}_{0}^{1}-\dot{x}_{0}^{2}\right\|
\end{aligned}
$$

from (3.54) in (3.55) we get

$$
\begin{aligned}
\left\|x_{\infty}\left(t, x_{0}^{1}\right)-x_{\infty}\left(t, x_{0}^{2}\right)\right\| \leq & E_{11}\left(E_{9} E_{3} E_{2} E_{10} E_{7}+E_{9} E_{5}\right)\left\|x_{0}^{1}-x_{0}^{2}\right\| \\
& +E_{11}\left(E_{9} E_{3} E_{2} E_{10} E_{8}+E_{9} E_{5}\right)\left\|\dot{x}_{0}^{1}-\dot{x}_{0}^{2}\right\|
\end{aligned}
$$

from (3.55) in (3.54) we get

$$
\begin{aligned}
\left\|\dot{x}_{\infty}\left(t, x_{0}^{1}\right)-\dot{x}_{\infty}\left(t, x_{0}^{2}\right)\right\| \leq & E_{12}\left(E_{10} E_{4} E_{1} E_{9} E_{5}+E_{10} E_{7}\right)\left\|x_{0}^{1}-x_{0}^{2}\right\| \\
& +E_{12}\left(E_{10} E_{4} E_{1} E_{9} E_{6}+E_{10} E_{8}\right)\left\|\dot{x}_{0}^{1}-\dot{x}_{0}^{2}\right\|
\end{aligned}
$$

Substituting (3.56) and (3.57) in (3.50). We get (3.49) .

\section{REFERENCE:}

1) Butris, R. N. Existence of a solution for system of second-order differential equation with Boundary conditions, Iraq, Mosul, J. Educ and sci vol (18) (1994).

2) Lazhar Bougoffa, A Class of Second Order Differential Equations with Nonlocal Boundary Conditions, Applied Mathematical Sciences, Vol. 1, (2007), no. 21, 1035 - 1039.

3) Martynuk, C. B. The investigation of solution for differential equations with boundary conditions. Ukrain. Kiev. Nauk. nonlinear problems in the theory of differential equations N0 2 (1991).

4) S. K. Ntouyas and P. Ch. Tsamatos, Existence and Uniqueness for Second Order Boundary value Problems, (Universty of loannina, Greece) Funkcialaj Ekvacioj 38(1995) 59-69.

5) Samoilenko, A. M. and Ronto, N. I. A numerical -analytic methods for investigations solutions with Boundary conditions Ukrain, Kiev, (1985).

6) Samoilenko, A. M. and Ronto, N. I. A numerical -analytic methods for investigations of periodic solutions, Ukrain, Kiev (1976).

7) Tadeusz Jankowski, Ordinary Differential Equation With Nonlinear Boundary Conditions, Georgian Mathematical Journal, Vol.9 (2002), Number 2, 287-294. 